\title{
Development of a Scalable Route for a Key Thiadiazole Building Block via Sequential Sandmeyer Bromination and Room-Temperature Suzuki-Miyaura Coupling

\author{
Gabriel Schäfer, ${ }^{*}$ Tony Fleischer, Muhamed Ahmetovic and Stefan Abele \\ Chemistry Process R\&D, Idorsia Pharmaceuticals Ltd., Hegenheimermattweg 91, \\ 4123 Allschwil, Switzerland \\ Email: gabriel.schaefer@idorsia.com
}

Supporting Information

Table of Contents

Page

1. Sandmeyer bromination optimization table

S2

2. Sandmeyer bromination process safety data

S3

3. NMR, DSC and HPLC Analysis of 1, 6 and 9-Li

S6

4. ${ }^{31} \mathrm{P}-\mathrm{NMR}$ spectrum of proposed Pd-Xantphos mono-oxide complex

S12 


\section{Sandmeyer bromination optimization table}

\begin{tabular}{llll} 
Nitrite & «Br» source & Solvent & Result \\
source & & & \\
\hline $\mathrm{NaNO}_{2}$ & $48 \%$ aq. $\mathrm{HBr}$ & $\mathrm{H}_{2} \mathrm{O}$ & Formation of brownish $\mathrm{Br}_{2}$ fog \& nasty reaction mixture. \\
& 0.1 eq. $\mathrm{CuBr}$ & & $10 \%$ conversion at $\mathrm{RT}$. \\
\hline $\mathrm{NaNO}_{2}$ & $48 \%$ aq. $\mathrm{HBr}$ & $\mathrm{H}_{2} \mathrm{O}$ & Similar conversion as with cat. CuBr \\
& 1.0 eq. $\mathrm{CuBr}$ & & \\
\hline tBu-ONO & $\mathrm{CuBr}(2.0$ eq.) & $\mathrm{MeCN}$ & Full conversion of $\mathrm{SM}$ at $60{ }^{\circ} \mathrm{C}$ (purity: ca. $50 \%$ a/a product) \\
\hline tBu-ONO & $\mathrm{CuBr} 2$ (1.5 eq.) & $\mathrm{MeCN}$ & Full conversion of $\mathrm{SM}$ at $2{ }^{\circ} \mathrm{C}$ (30 min). Clean reaction (ca.
\end{tabular}


All reactions performed on $500 \mathrm{mg}$ scale. Nitrite sources: 1.5 eq.; Solvent: 10 vol. 


\section{Sandmeyer bromination process safety data}

Reaction performed according to general procedure described in the experimental section on 10 g scale in a $400 \mathrm{~mL}$ reaction calorimeter with overhead stirred (Systag FlexyCUBE).

Dosing time of tert-butyl nitrite: $30 \mathrm{~min}$
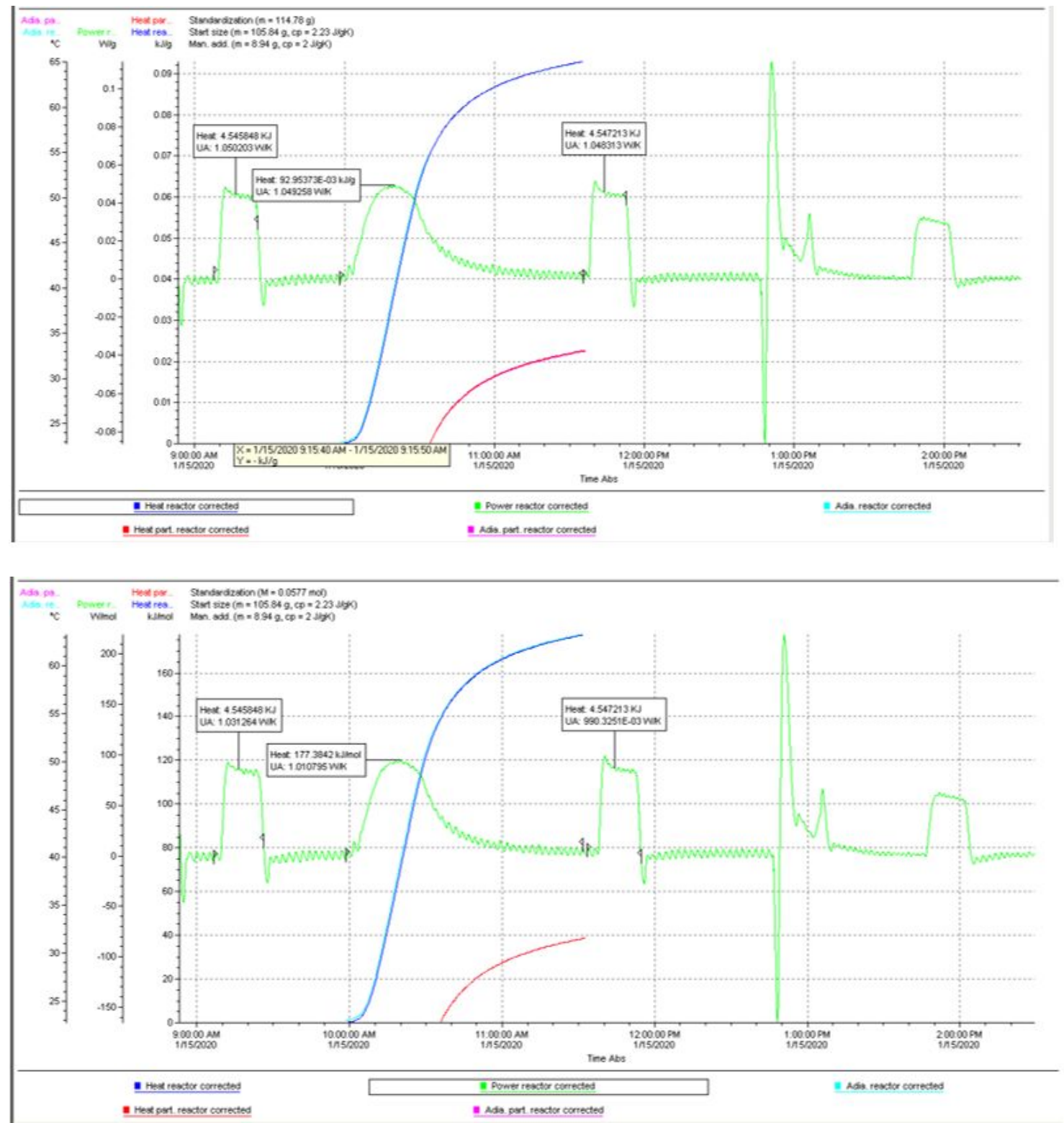

tert-butyl nitrite addition: 
Observed Main Energy release: $\quad Q R=92.9 \mathrm{~kJ} / \mathrm{kg}$

$\mathrm{HR}=177 \mathrm{~kJ} / \mathrm{mol}$

$\Delta \mathrm{Tad}=\mathrm{QR} / \mathrm{Cp}: \quad \sim 41.65^{\circ} \mathrm{C}$

$\mathrm{MTSR}=64.65^{\circ} \mathrm{C}\left(\mathrm{MTSR}=\mathrm{Tp}+\Delta \mathrm{Tad}=23^{\circ} \mathrm{C}+41.65=64.65^{\circ} \mathrm{C}\right)$

Reaction in Thermal Scanning Unit (TSU) from HEL

Reaction mixture after dosing of tert-butyl nitrite was subjected into $10 \mathrm{~mL}$ sample cell from $\mathrm{HEL}$ and TSU run was performed. Measured was reaction exotherm and generated pressure release during the event.

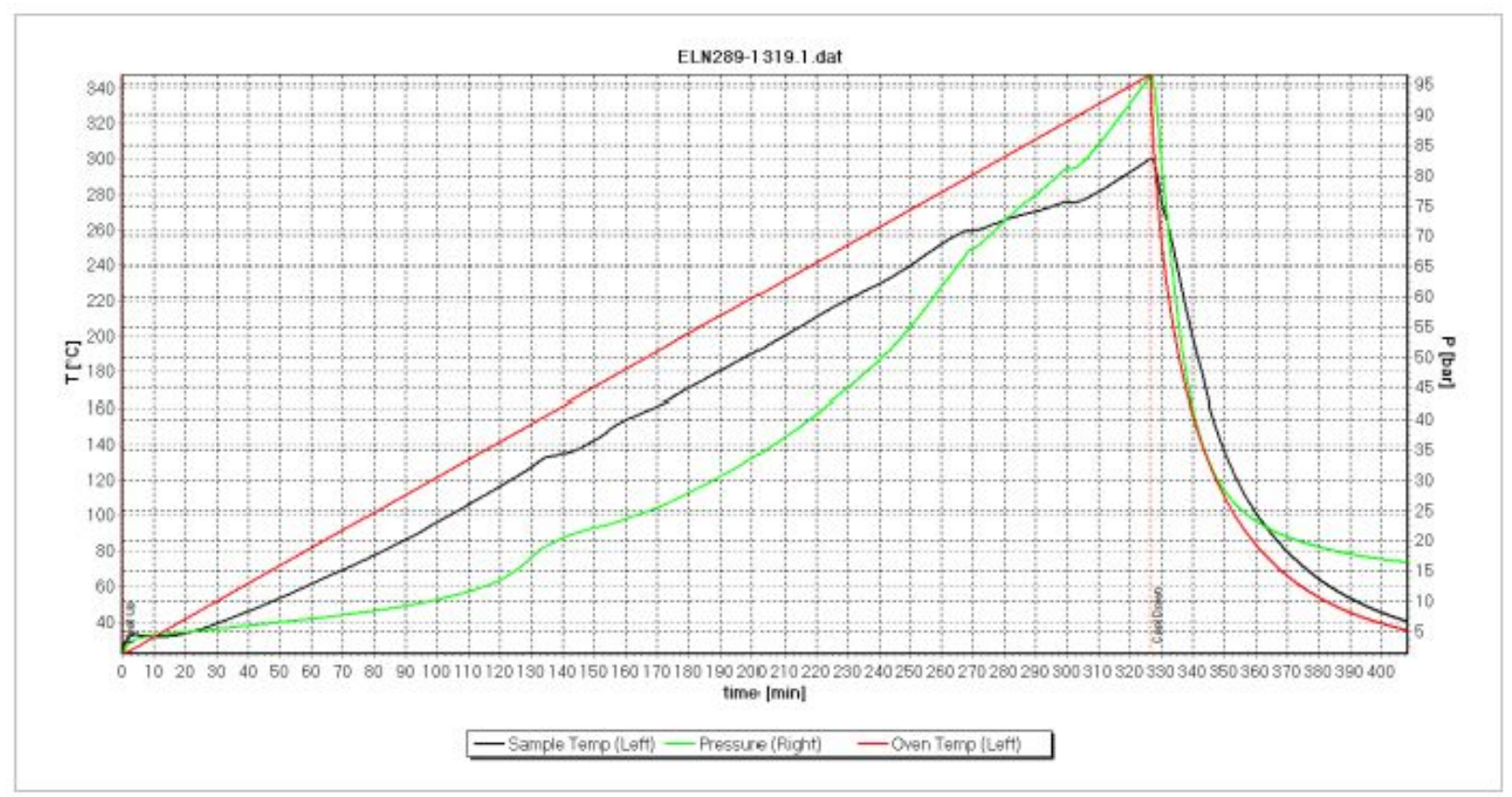




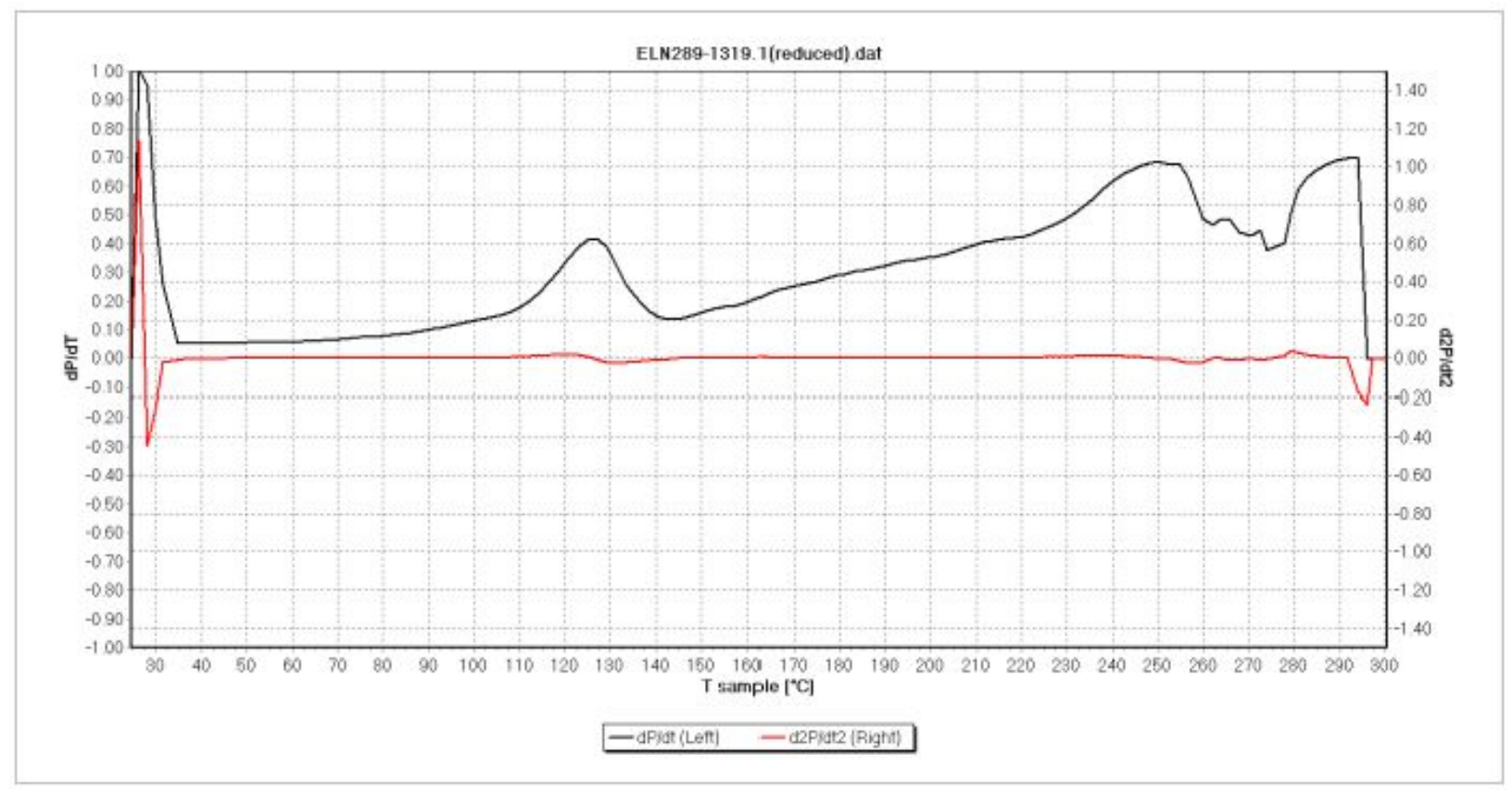

TsU runs show gas release of reaction mixture above $120^{\circ} \mathrm{C}$, reaction is run ca. $100{ }^{\circ} \mathrm{C}$ below this temperature.

DSC of tert-butyl nitrite

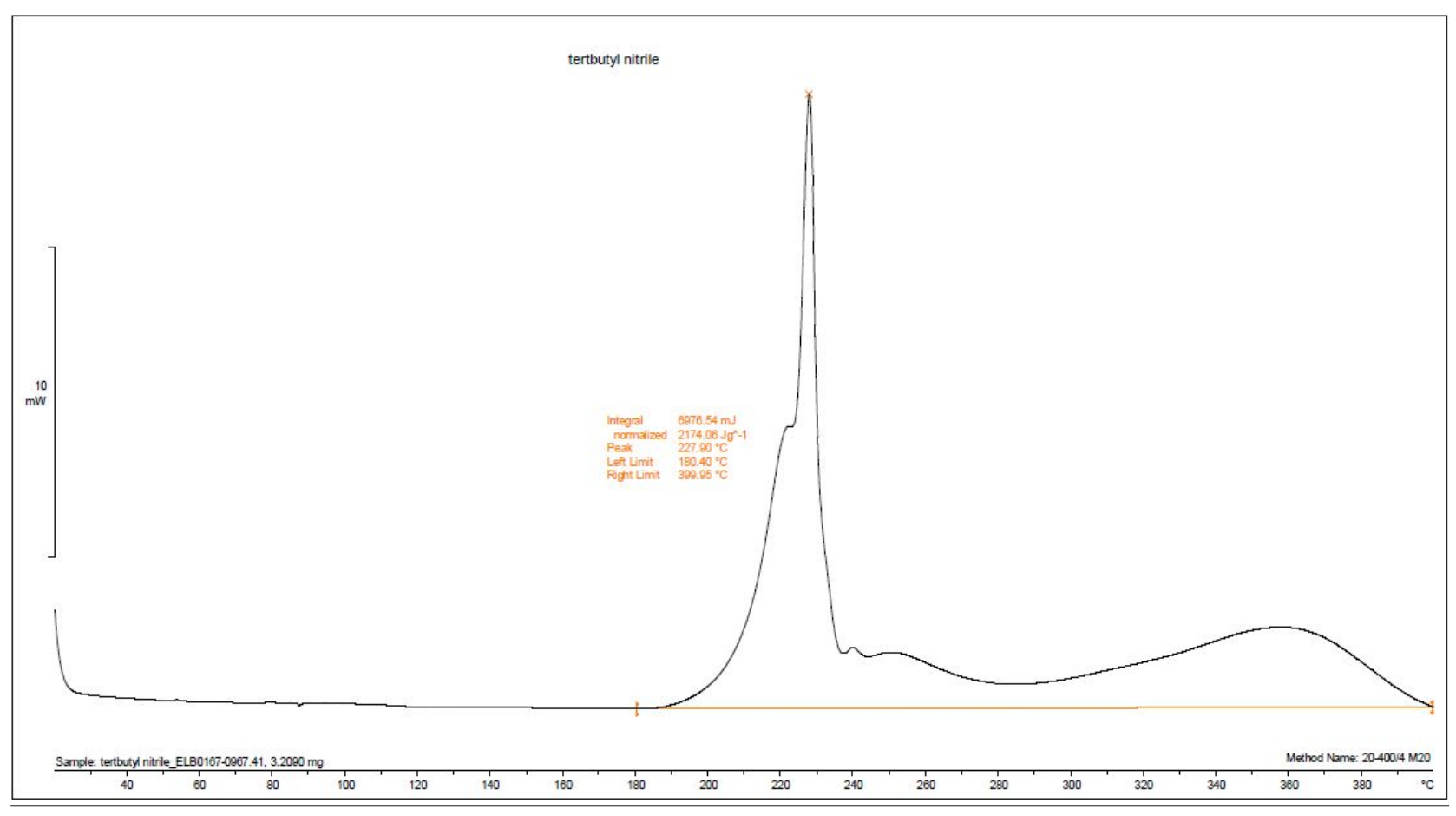


3. NMR, DSC and HPLC Analysis of 1, 6 and 9-Li

Ethyl 5-(2,4-difluorophenyl)-1,3,4-thiadiazole-2-carboxylate (1) 

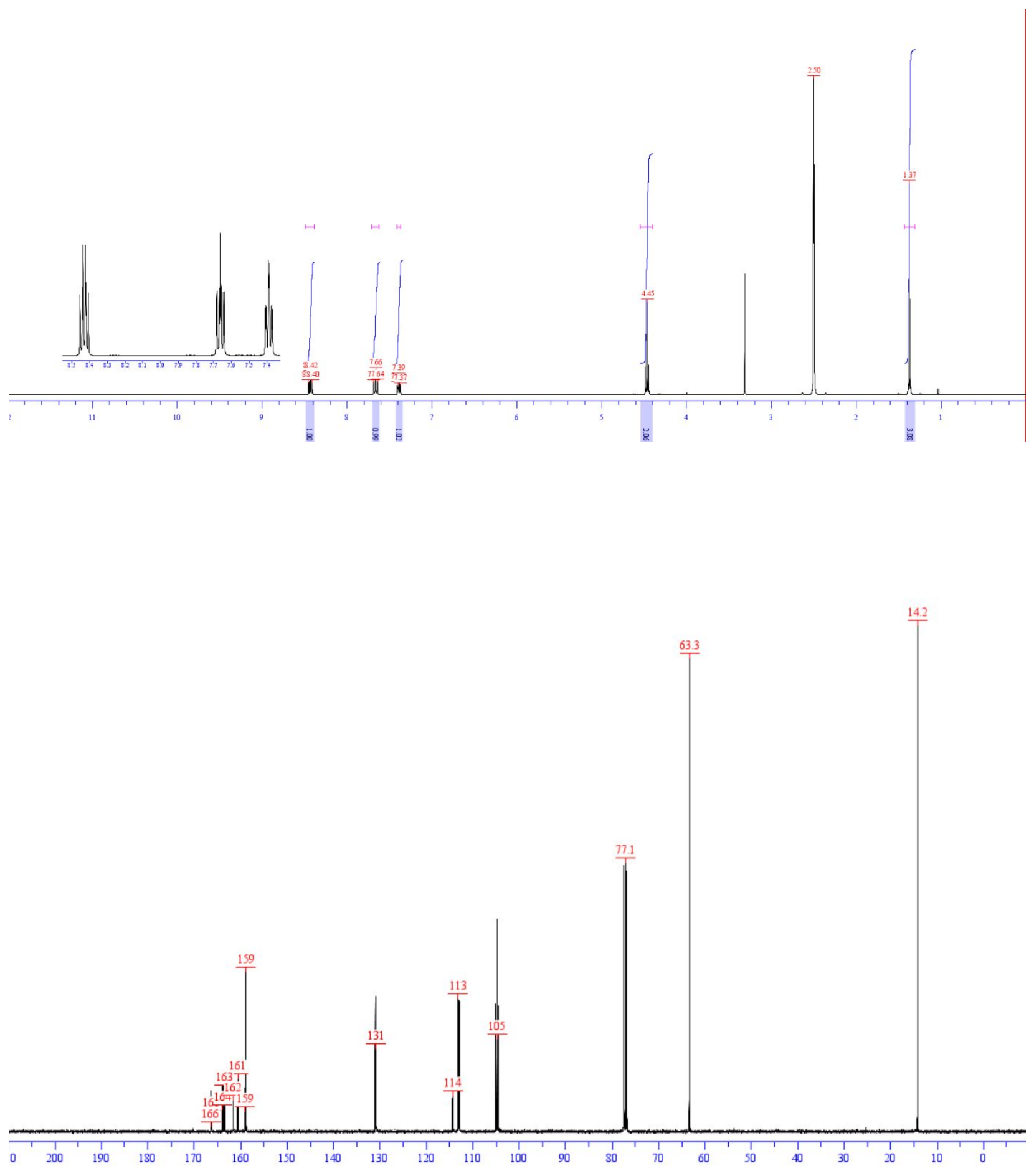


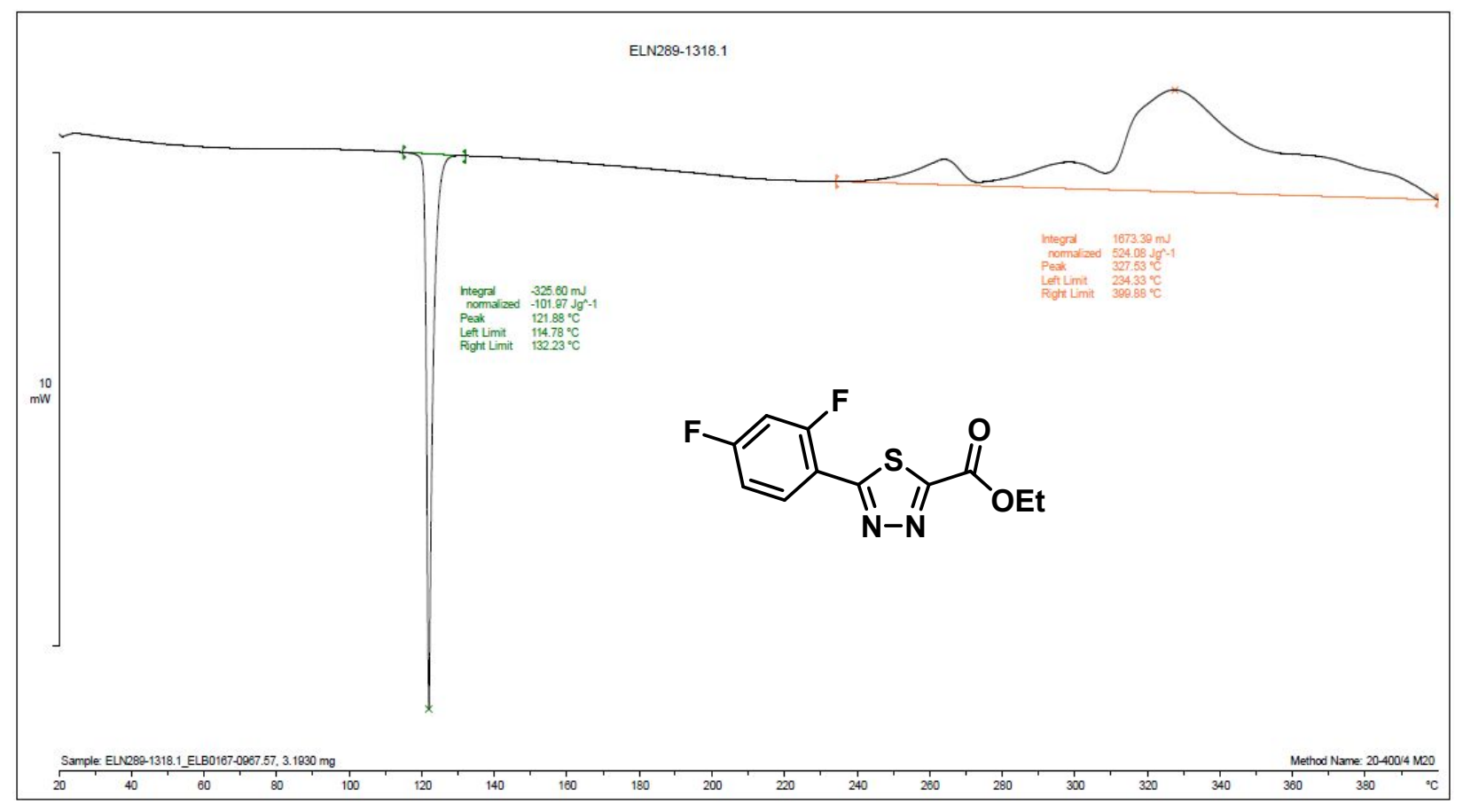

\section{HPLC trace of product (1) from $200 \mathrm{~g}$ run with charcoal-treated starting material}
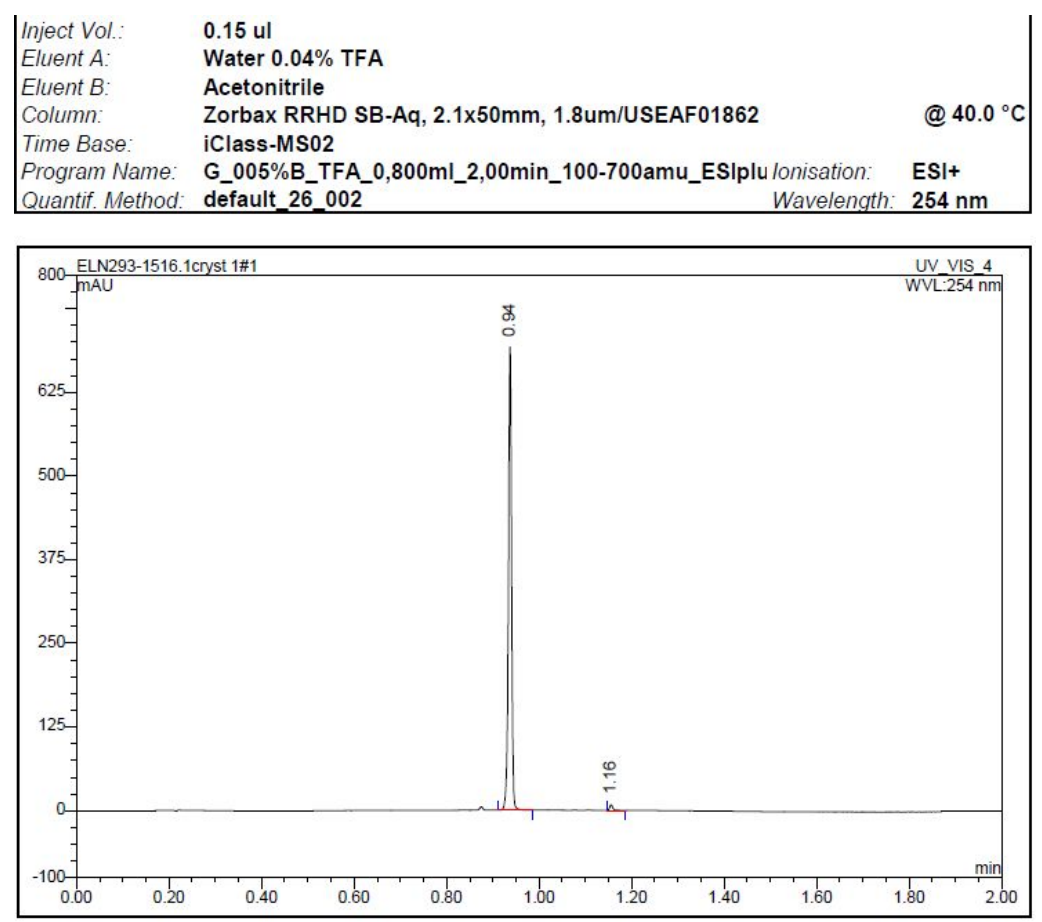

\begin{tabular}{cccccccc}
\hline $\begin{array}{c}\text { Peak No. } \\
\text { UV_VIS_4 }\end{array}$ & $\begin{array}{c}\text { Ret.Time } \\
\text { UV_VIS_4 } \\
\text { min }\end{array}$ & $\begin{array}{c}\text { Area } \\
\text { UV_VIS_4 }\end{array}$ & $\begin{array}{c}\text { Rel.Area } \\
\text { UV_VIS_4 } \\
\text { \% }\end{array}$ & $\begin{array}{c}\text { Mass 01 } \\
\text { UV_VIS_4 } \\
\text { amu }\end{array}$ & $\begin{array}{c}\text { Mass 02 } \\
\text { UV_VIS_4 } \\
\text { amu }\end{array}$ & $\begin{array}{c}\text { Mass 03 } \\
\text { UV_VIS_4 } \\
\text { amu }\end{array}$ & $\begin{array}{c}\text { UV Match } \\
\text { UV_VIS_4 }\end{array}$ \\
\hline 1 & 0.94 & 5454 & 98.8 & 271.12 & 272.14 & 312.10 & 989 \\
2 & 1.16 & 65 & 1.2 & 309.04 & 270.97 & 142.21 & 1000 \\
\hline Total: & \multicolumn{7}{c}{100.0} \\
\hline
\end{tabular}


Ethyl 5-bromo-1,3,4-thiadiazole-2-carboxylate (6)
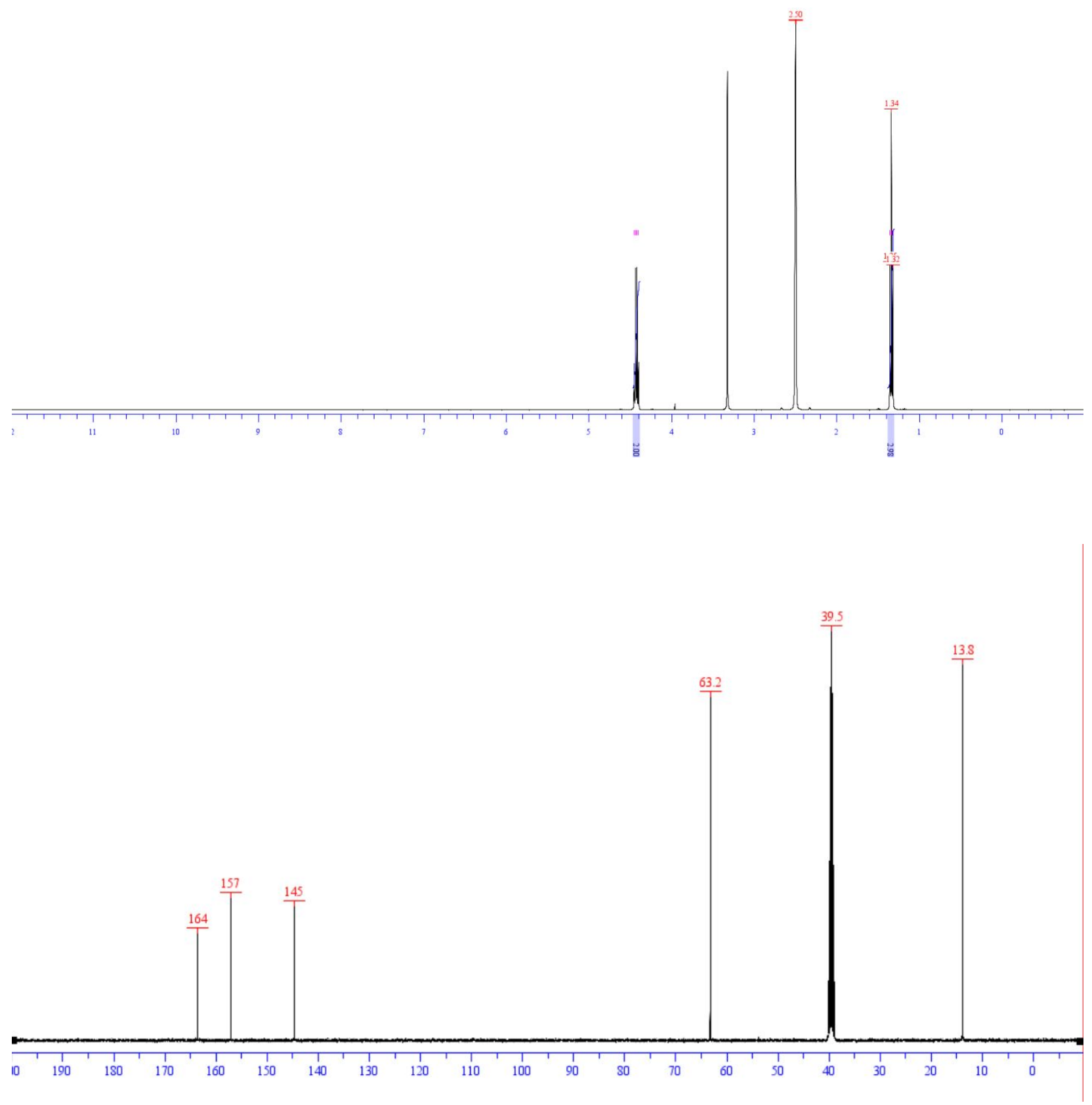


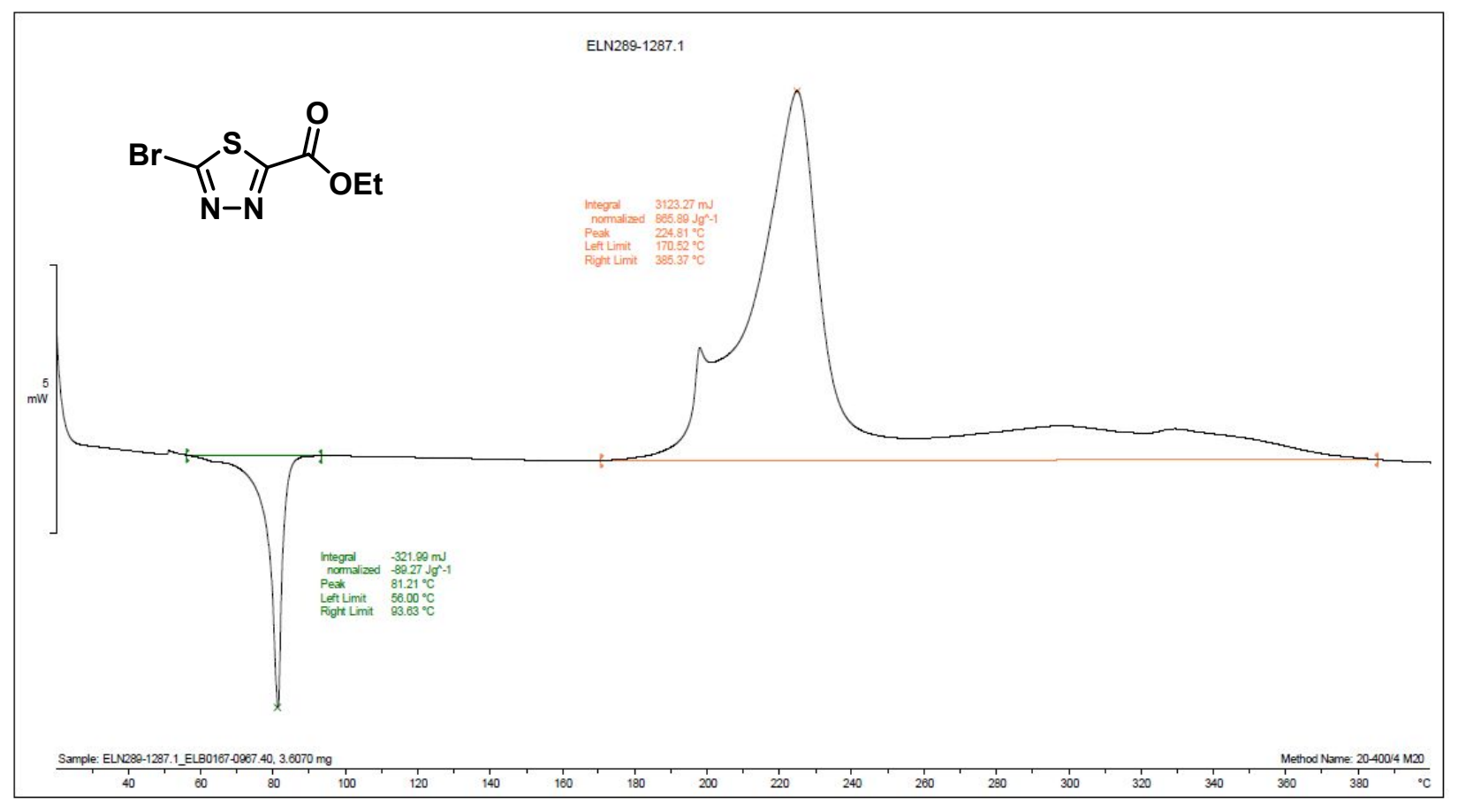

HPLC trace of product (6) from $250 \mathrm{~g}$ run with charcoal treatment 


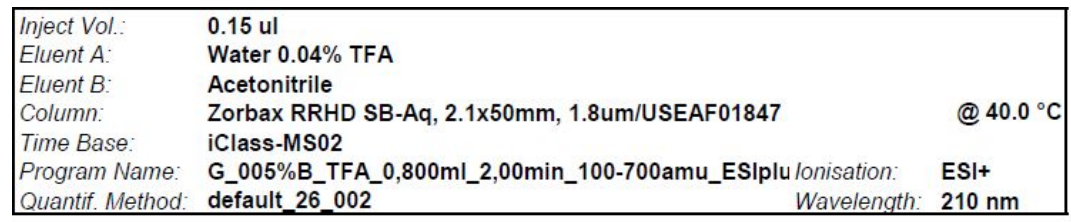

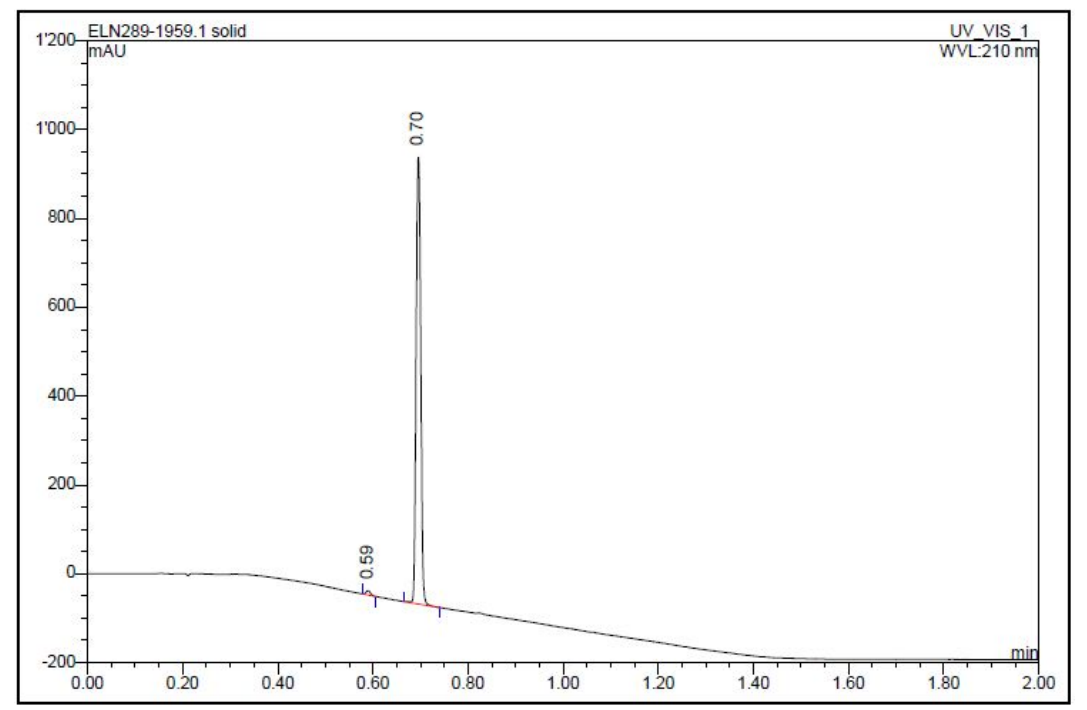

\begin{tabular}{cccccccc}
\hline $\begin{array}{c}\text { Peak No. } \\
\text { UV_VIS_1 }\end{array}$ & $\begin{array}{c}\text { Ret.Time } \\
\text { UV_VIS_1 } \\
\text { min }\end{array}$ & $\begin{array}{c}\text { Area } \\
\text { UV_VIS__1 }\end{array}$ & $\begin{array}{c}\text { Rel.Area } \\
\text { UV_VIS_1 } \\
\text { \% }\end{array}$ & $\begin{array}{c}\text { Mass 01 } \\
\text { UV_VIS_1 } \\
\text { amu }\end{array}$ & $\begin{array}{c}\text { Mass 02 } \\
\text { UV_VIS_1 } \\
\text { amu }\end{array}$ & $\begin{array}{c}\text { Mass 03 } \\
\text { UV_VIS_1 } \\
\text { amu }\end{array}$ & $\begin{array}{c}\text { UV Match } \\
\text { UV_VIS_1 }\end{array}$ \\
\hline 1 & 0.59 & 102 & 0.9 & 142.21 & 143.27 & 268.01 & 1000 \\
2 & 0.70 & 11302 & 99.1 & 280.03 & 278.06 & 239.06 & 999 \\
\hline
\end{tabular}

Lithium 5-(2,4-difluorophenyl)-1,3,4-thiadiazole-2-carboxylate (9-Li)

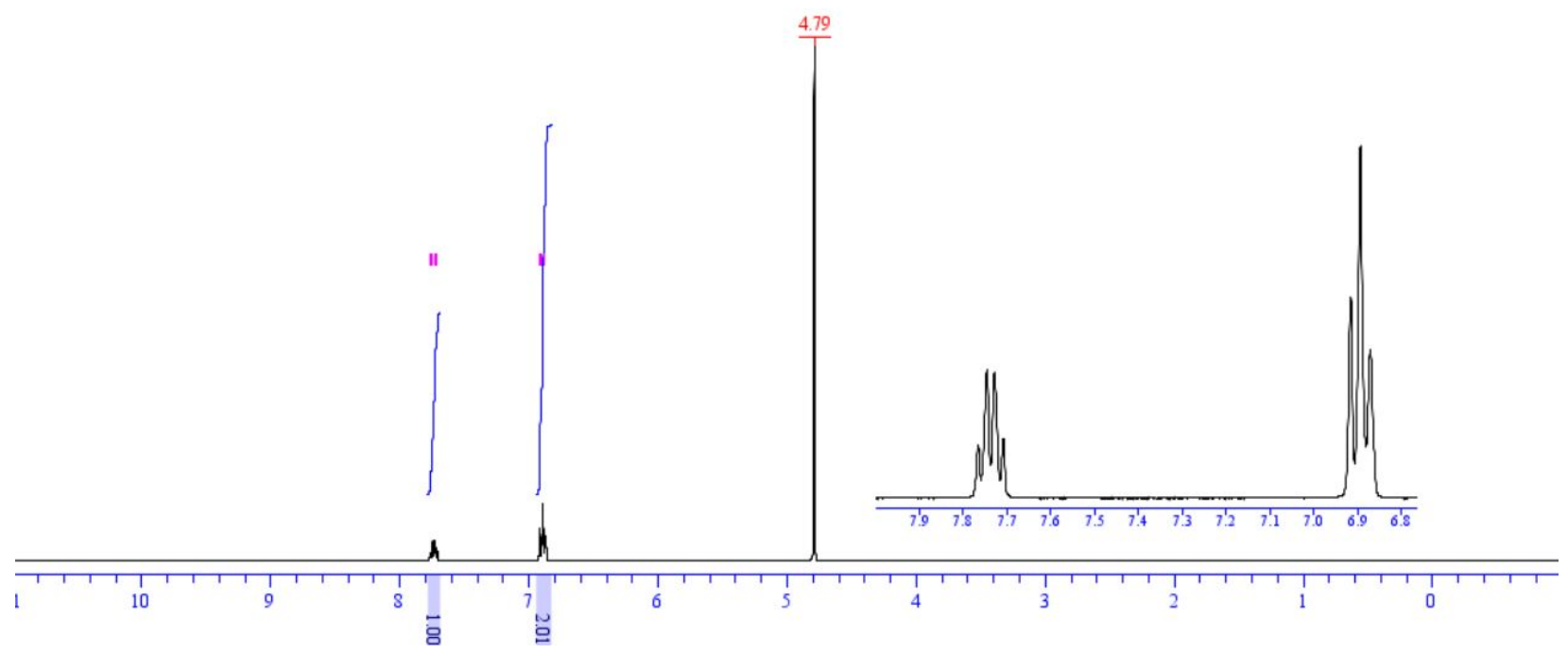




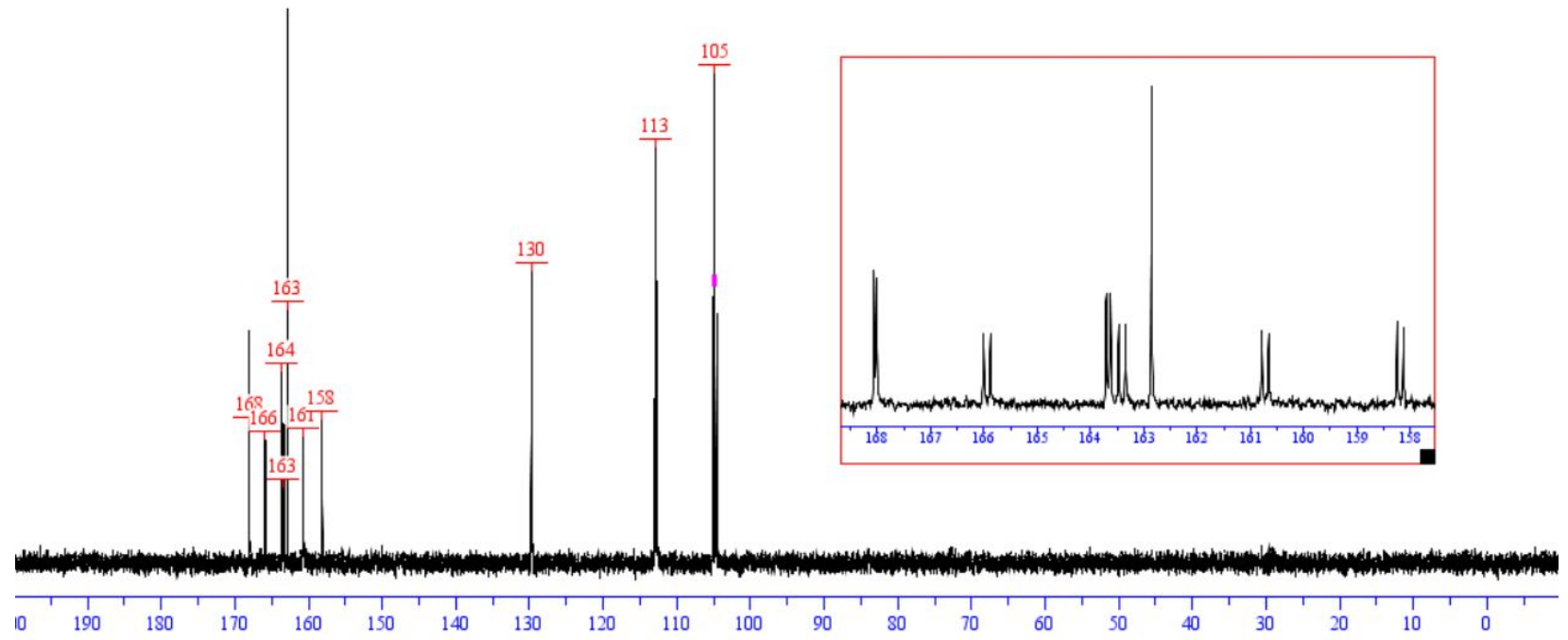




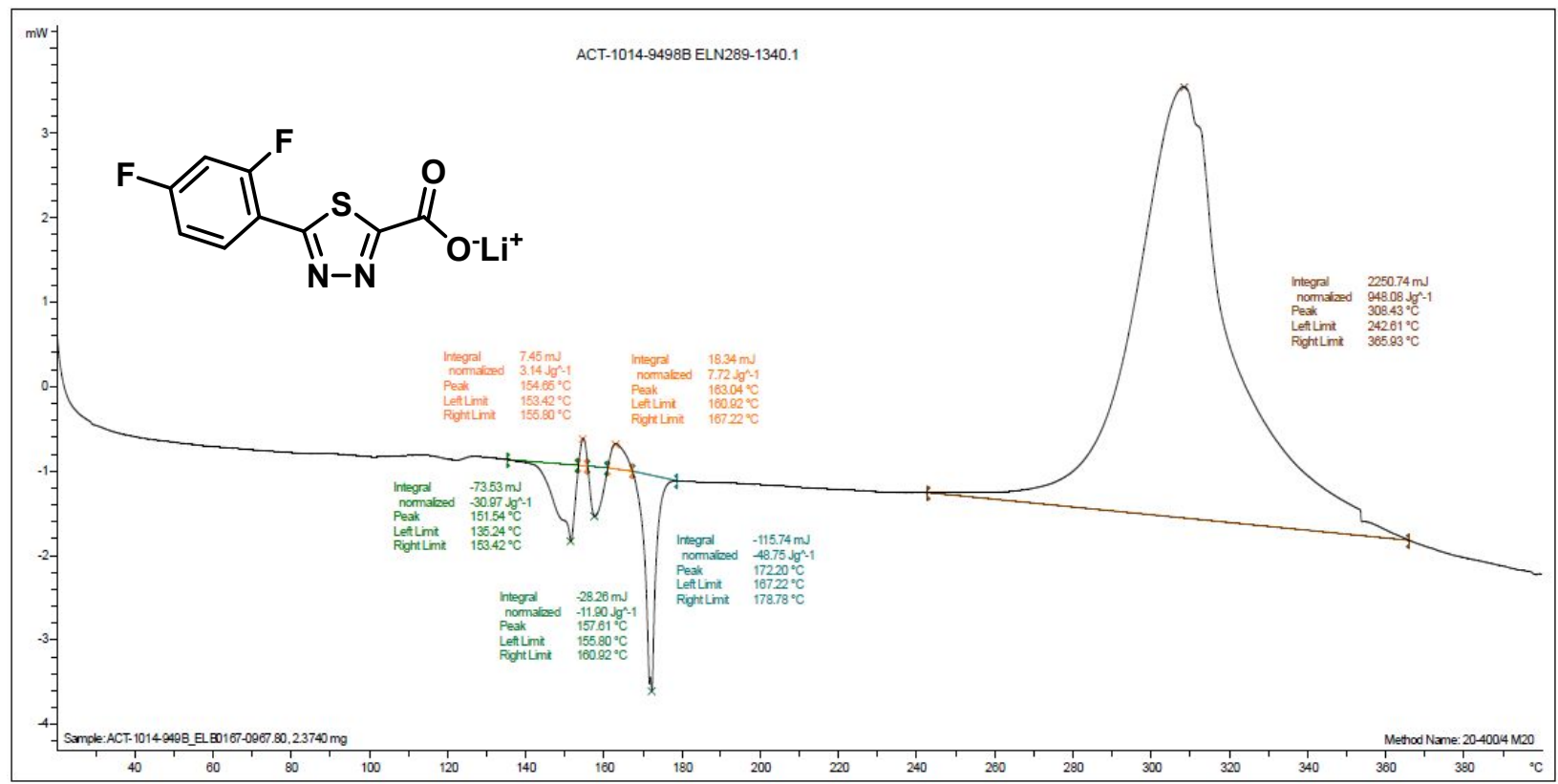

\section{HPLC trace of product (9-Li) from $250 \mathrm{~g}$ run with charcoal treatment}

\begin{tabular}{|c|c|c|}
\hline $\begin{array}{l}\text { Inject Vol:: } \\
\text { Eluent A: } \\
\text { Eluent B: } \\
\text { Column: } \\
\text { Time Base: } \\
\text { Program Name: } \\
\text { Quantif. Method: }\end{array}$ & 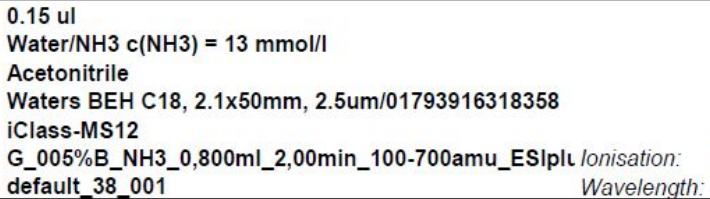 & $\begin{array}{l}\text { @ } 40.0^{\circ} \mathrm{C} \\
\mathrm{ESI+} \\
210 \mathrm{~nm}\end{array}$ \\
\hline
\end{tabular}

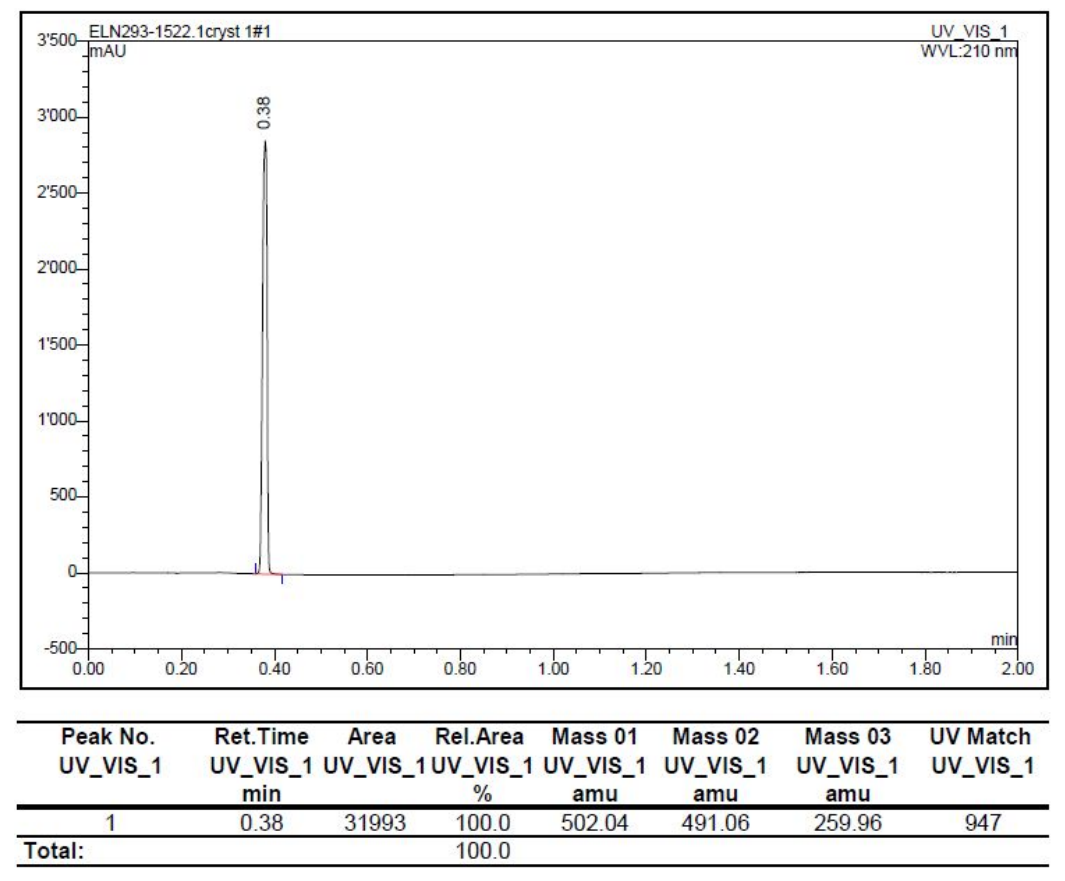




\section{4. ${ }^{31} \mathrm{P}-\mathrm{NMR}$ spectrum of proposed Pd-Xantphos mono-oxide complex}

Under N2-atmosphere, a $4 \mathrm{~mL}$ screw-cap vial was charged with $\mathrm{Pd}(\mathrm{OAc})_{2}(9.5 \mathrm{mg})$ and Xantphos $(24.4 \mathrm{mg})$. Then, toluene-d8 $(1.0 \mathrm{~mL})$ was added and the red solution stirred for 30 min at rt. Then, the aryl bromide (100 mg), NMM (93.6 microliter) and $\mathrm{D}_{2} \mathrm{O}(0.5 \mathrm{~mL})$ were added and the biphasic mixture stirred again for $60 \mathrm{~min}$. The stirring was stopped, and the toluene layer transferred into a NMR-tube (performed under air, no $\mathrm{N}_{2}$-purge of tube) and analyzed by ${ }^{31} \mathrm{P}$ NMR.

Note: The isolation and full characterization of the Pd-Xantphos mono-oxide complex has so far not been achieved.

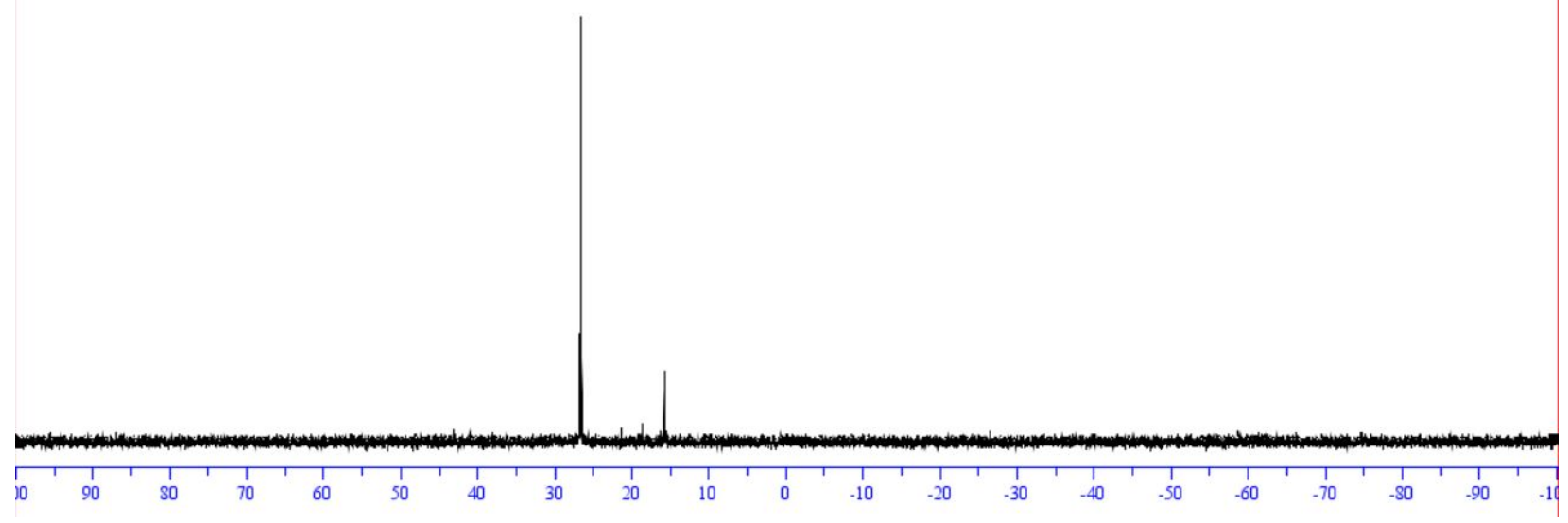




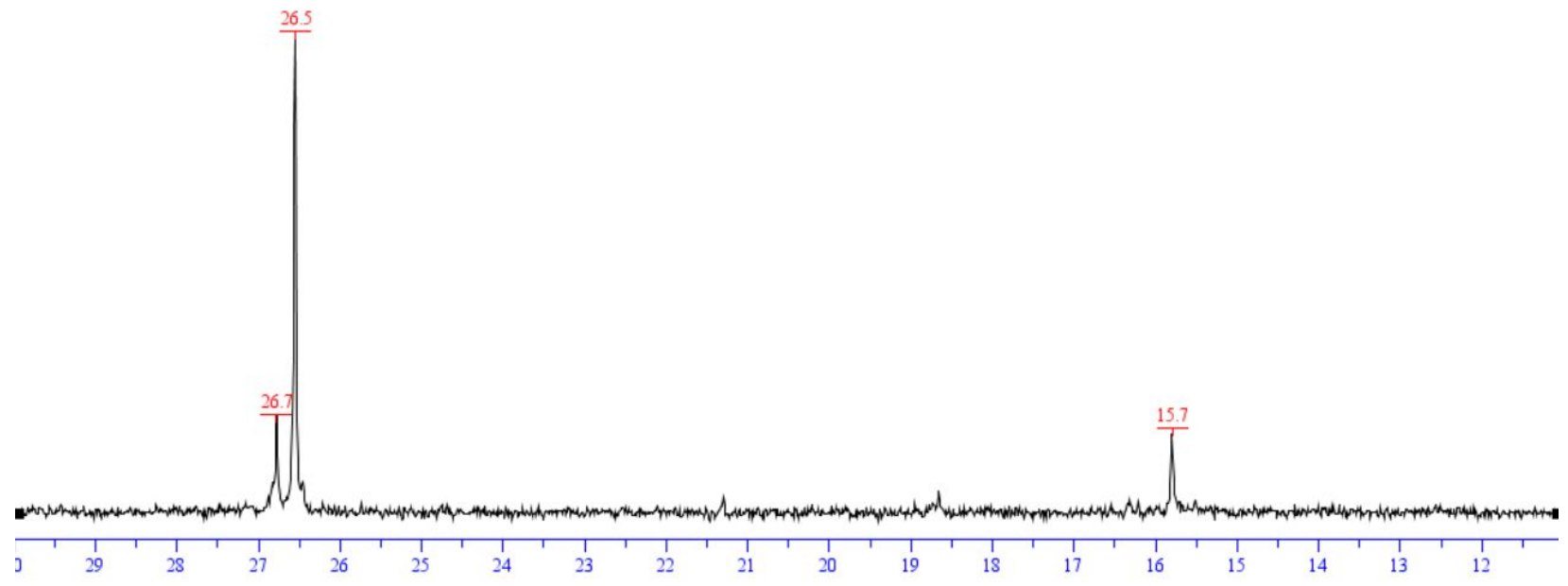

